\title{
Sustained reversed magnetic shear mode of operation in the HL-2A tokamak
}

\author{
Qingdi Gao, R.V. Budny*, Jinhua Zhang, Fangzhu Li, Yiming Jiao \\ Southwestern Institute of Physics, \\ Chengdu, China \\ * Princeton Plasma Physics Laboratory, \\ Princeton University, Princeton, New Jersey, United States of America
}

\begin{abstract}
Realistic reversed magnetic shear (RS) discharges for the HL-2A tokamak are simulated with the TRANSP code. Early NBI heating achieves reversed shear (RS) configurations characterized by highly peaked pressure profiles and a narrow shear reversal region. To avoid excessive peaks in the pressure, and to sustain RS operation towards steady state, LHCD is used to control the current profile. The combined LH and bootstrap current create a quasi-stationary RS discharge with $r_{\min } / a \approx 0.6$ and $q_{\min } \approx 2.8$. A self-consistent internal transport barrier is produced, and the RS configuration is established with a wider shear reversal region and broader pressure profile that is favourable to achieving the high beta limit.
\end{abstract}

\section{Introduction}

HL-2A is a new tokamak under construction at SWIP (Southwestern Institute of Physics), Chengdu. One of the HL-2A objectives is to study enhanced core plasma confinement by profile control. Studies $[1,2]$ on the optimization of current density profile suggest that reversed magnetic shear (RS) is desirable for confinement, stability and bootstrap alignment. In some other tokamaks such as TFTR [3], DIII-D [4], JT-60U [5] and Tore Supra [6], RS plasmas develop internal transport barriers (ITBs) that produce improved central confinement. Discharges with ITBs have also been established with optimized magnetic shear (OS) in JET $[7,8]$ and ASDEX Upgrade [9]. The good central confinement can lead to peaked pressure profiles, which would generate a strong off-axis bootstrap current and sustain the hollow current density profile. This scenario is an attractive candidate for steady state tokamak operation. However, the RS discharges established in the early experiments were usually transient in nature due to the development of MHD instabilities.

The experimental observation of greatly reduced transport in RS plasmas provides a strong motivation to further explore current profile control by which optimized RS operation may be established and maintained for timescales beyond the characteristic current diffusion time. Recently in the JT-60U tokamak [10], quasi-steady sustainment of
RS discharges has been achieved by step-down of beam injection power or the use of a current profile control scheme with LHCD applied consistently with a bootstrap current profile. In Tore Supra discharges [6], stable and stationary states with reversed magnetic shear have been achieved with LHCD during reduced magnetic operation. In the HL-2A tokamak, the various schemes of auxiliary heating and current drive including NBI (3 MW), ICRH (1 MW), LHCD (2.5 MW) and ECRH (0.5 MW) combined with the device flexibility offer the opportunity to optimize the current profile. In this article we model realistic RS discharges for HL-2A using the TRANSP code to find favourable scenarios for RS operation. Subsequent to establishing self-consistent RS equilibria with NBI, we optimize the plasma configuration using LHCD.

The article is organized as follows. In Section 2, an outline of HL-2A is given. Section 3 contains a TRANSP simulation of the RS discharge formed with NBI in HL-2A. In Section 4, the current profile control with LHCD is discussed and the sustained RS operation by use of non-inductive current drive applied consistently with bootstrap current is analysed. A summary is presented in Section 5.

\section{Outline of the HL-2A tokamak}

The HL-2A tokamak, as the first step of the HL-2 project [11], is under construction using the ASDEX 
Table 1. The main parameters of HL-2A

\begin{tabular}{llll}
\hline Major radius $R(\mathrm{~m})$ & 1.64 & 1.75 & 1.67 \\
Minor radius $a(\mathrm{~m})$ & 0.4 & 0.38 & 0.32 \\
Aspect ratio $A$ & 4.1 & 4.6 & 5.2 \\
Elongation $k_{x}$ & 1.0 & 1.1 & 1.3 \\
Triangularity $\delta_{x}$ & 0.0 & 0.44 & 0.5 \\
Toroidal field $B_{T}(\mathrm{~T})$ & 2.8 & 2.8 & 2.8 \\
Plasma current $I_{p}(\mathrm{MA})$ & 0.48 & 0.45 & 0.45 \\
$q_{\psi}$ (at separatrix) & 3.26 & 3.46 & 3.50 \\
Current flat-top $t_{f}(\mathrm{~s})$ & $3.0-5.0$ & 3.0 & 3.0 \\
Magnetic geometry & Fig. $1(\mathrm{a})$ & Fig. $1(\mathrm{~b})$ & Fig. $1(\mathrm{c})$ \\
Auxiliary heating power $(\mathrm{MW})$ & & & \\
$\quad$ NBI (energy $E=40 \mathrm{keV})$ & $3.0(2.5)$ & 3.0 & 3.0 \\
$\quad$ ICRH (frequency $f=38 \mathrm{MHz})$ & $1.0(2.0)$ & 1.0 & 0.5 \\
$\quad$ ECRH (frequency $f=73 \mathrm{GHz})$ & 0.5 & 0.5 & 2.5 \\
$\quad$ LHCD (frequency $f=2.45 \mathrm{GHz})$ & 2.5 & 2.5 & 16 \\
Number of $B_{T}$ coils & 16 & 16 & 1.0 \\
$B_{t}$ ripple at outer plasma edge $(\%)$ & 1.1 & 2.1 & \\
\hline
\end{tabular}
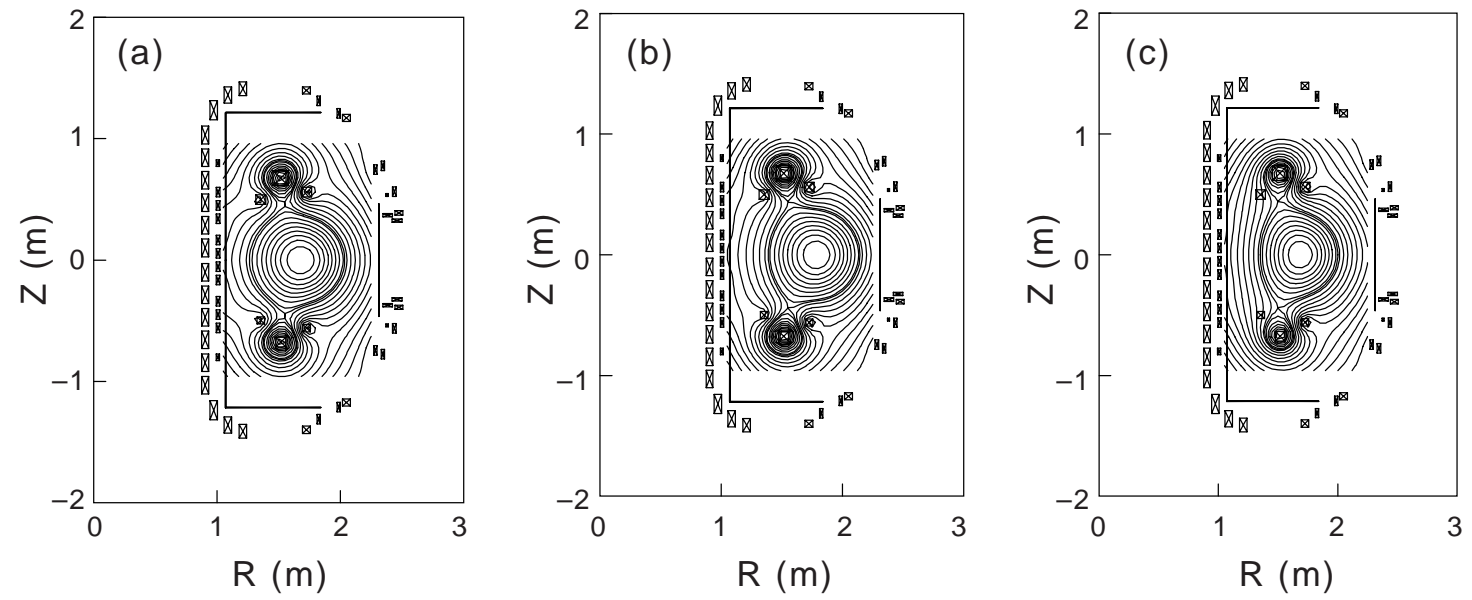

Figure 1. Magnetic geometry of HL-2A with (a) a circular cross-section, (b) the X point moving inwards and (c) modest shaping.

load assembly. The main objectives of HL-2A are to produce more adaptable divertor configurations to study energy exhaust and impurity control (the first divertor tokamak plasma in China), and to study enhanced core plasma confinement by profile control and moderate plasma shaping. Furthermore, it is essential to gaining experience for the building and operation of a much more complicated advanced tokamak (HL-2). The main parameters of HL-2A are given in Table 1 (also see Fig. 1).

\section{Shear reversal formation with NBI}

The time dependent TRANSP plasma analysis code, with capabilities to model complex tokamak discharges [12], is used to simulate RS operation in HL-2A. The TRANSP analysis code is being used extensively to analyse experimental results from many tokamaks, such as TFTR, JET, DIIID, TEXTOR, Tore Supra and Alcator C-Mod. The widespread use of TRANSP increases its reliability 


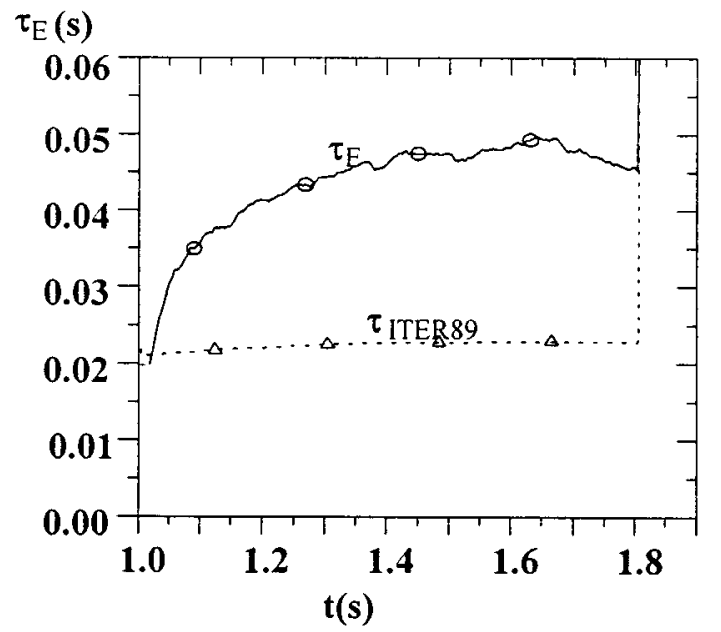

Figure 2. Energy confinement time $\tau_{E}$ and $\tau_{I T E R 89}$ versus time. Reversed shear begins at $t=1.0 \mathrm{~s}$.

and facilitates comparison of results from different experiments. In addition to the analysis of tokamak plasmas using measured parameters, TRANSP has a limited predictive capability. The plasma current and $q$ profile evolutions can be computed from the poloidal field diffusion equation.

The first discharge discussed is a deuterium discharge with the plasma current ramped up to $265 \mathrm{kA}$ at $0.3 \mathrm{~s}$. The electron density is assumed to be peaked, as in the case of many tokamak plasmas with enhanced confinement, such as the supershots and enhanced reversed shear (ERS) plasmas in TFTR. The central electron density $n_{e}(0)$ increases from $2.7 \times 10^{19}$ to $1.1 \times 10^{20} \mathrm{~m}^{-3}$, and the peaking of the electron density $n_{e}(0) /\left\langle n_{e}\right\rangle$ increases from 1.7 to $3.4\left(\left\langle n_{e}\right\rangle\right.$ is the volume averaged density). In HL-2A, a closed divertor configuration will be combined with divertor pumping [11] to effectively screen the
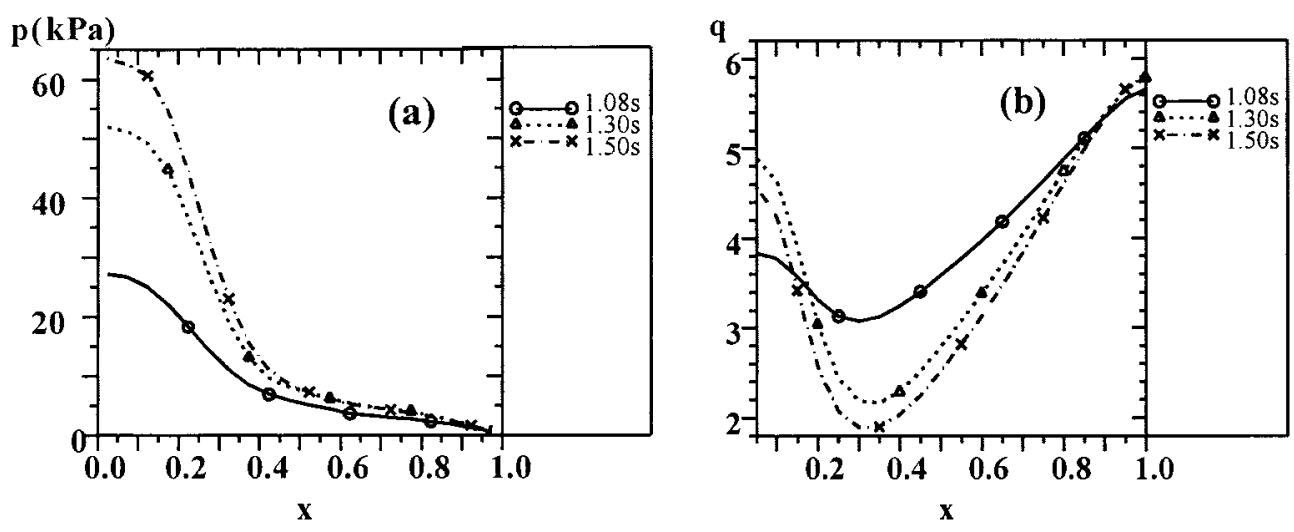

Figure 3. Profiles of (a) thermal pressure and (b) $q$ at $t=1.08,1.30$ and $1.50 \mathrm{~s}$ for the RS discharge formed with NBI.

Nuclear Fusion, Vol. 40, No. 11 (2000)

1899 
internal inductance $l_{i}=0.65$. The poloidal beta and normalized beta are, respectively, $\beta_{p}=1.21$ and $\beta_{n}=1.45$ with a pressure peaking factor of $p(0) /\langle p\rangle=5.1$, where $\langle p\rangle$ stands for volume averaged pressure.

(b) The case at $t=1.30 \mathrm{~s}$ is a configuration with higher bootstrap current. The plasma current is the same as that in the previous case with more than half of the current driven from the bootstrap effect $\left(f_{B S}=0.51\right)$ and $\sim 15 \%$ driven by the neutral beam $\left(I_{N B}=33 \mathrm{kA}\right)$. The current profile is more hollow with $q_{0}=4.84$, $q_{\text {min }}=2.14, q_{\psi}=5.76$ and $l_{i}=0.79$. The shear reversal region is a little wider than that in the previous case, $x_{\text {min }} \approx 0.32$. Since the plasma density and electron temperature are increased, the beta values are higher, $\beta_{p}=1.65, \beta_{n}=1.74$ and $p(0) /\langle p\rangle=5.5$.

These RS configurations have highly peaked pressure profiles. However, excessive peaking of the pressure can lead to instability of low- $n$ modes, and many RS discharges end disruptively at rather modest beta. For example, the disruption of RS discharges in TFTR is identified to be caused by an ideal $n=1$ instability, an infernal/kink mode [13], the bursting MHD activity observed near the disruption boundary of negative central shear (NCS) discharges in DIII-D is due to resistive interchange modes [14], and in the OS discharges in JET a global $n=1$ ideal mode is observed to be unstable at the time the disruption precursor occurs [15]. The theoretical investigations on the MHD operational limits of the RS tokamaks [16-18] also conclude that the optimal pressure profiles are never strongly peaking.

In addition, these RS configurations for HL-2A have a rather narrow negative shear region with the minimum $q, q_{\min }$ evolving towards $q_{\min }<2$. The RS experiments in tokamaks show that when the value of $q_{\text {min }}$ decreases with time, the hard collapse or disruption occurs when $q_{\min }$ becomes either $3,2.5$ or 2 [19].

The HL-2A RS discharge discussed above has been analysed with respect to several MHD instability modes including low- $n$ MHD modes, ballooning modes and resistive interchange modes [20]. According to the stability analysis, the RS equilibria with highly peaked pressure profiles are not stable to several MHD modes; in particular the low- $n$ internal modes located in the low shear region are unstable, development of which would terminate the RS discharge disruptively.

\section{Sustained RS mode of operation}

\subsection{Calculation of the LH wave driven current}

In order to sustain the RS discharges towards steady state, off-axis current drive with LH waves is used for current profile control, and we describe here a simulation for RS discharges with LHCD to predict the plasma configuration and the plasma behaviour.

The LH wave power spectrum radiated by a multijunction launcher $(2 \times 12)$ is calculated with the Brambilla coupling theory [21]. By tuning the phasing between wave guides, spectra with central parallel refractive index $n_{\| 0}=1.64,2.48,3.34$ and 4.22 are yielded, corresponding to phasings $\Delta \phi=60,90$, 120 and $150^{\circ}$, respectively.

The LHCD calculation [22] utilizes a toroidal ray tracing algorithm for the wave propagation and a parallel velocity Fokker-Planck calculation for the interaction of waves and particles. The ray information, with an assumption of low power and linear damping, is used to estimate the quasi-linear flux surface averaged diffusion coefficient. Then an electron velocity distribution function is obtained by solving a 1-D Fokker-Planck equation with the effects of the local electric field neglected. The power level is raised gradually while the distribution function, power deposition and quasi-linear diffusion coefficient are iteratively recomputed. After this, the current driven on each flux surface is calculated according to [23]

$j_{L H}=\frac{-e n_{e}}{\nu_{r}} \int d v_{\|} D_{q l}\left(v_{\|}\right) \frac{\partial f_{e}\left(v_{\|}\right)}{\partial v_{\|}} \frac{\partial W_{s}(u)}{\partial u}$

with the following notation used:

$D_{q l}\left(v_{\|}\right)=\frac{\pi}{2}\left(\frac{e}{m_{e}}\right)^{2} E_{\|}^{2} \delta\left(\omega-k_{\|} v_{\|}\right)$

$\nu_{r}=(\ln \Lambda) n_{e} e^{4} / 4 \pi \varepsilon_{0}^{2} m_{e}^{2} /\left|v_{r}\right|^{3}, \quad u=v_{\|} / v_{r}$

where

$v_{r}=-\operatorname{sgn}\left(e E_{D C}\right) \sqrt{n_{e} e^{4} \ln \Lambda / 4 \pi \varepsilon_{0}^{2} m_{e}\left|e E_{D C}\right|}$.

The key quantity is $W_{s}(u)$, the energy (normalized to $\left.m_{e} v_{r}^{2} / 2\right)$ imparted to the electric field $E_{D C}$ by an electron as it slows down. The local electric field $E_{D C}$ is either prescribed for a static simulation or supplied by TRANSP as part of the iteration. For a very small electric field, $W_{s}(u)$ can be represented in 
a power series, from the first two terms of which is found

$$
\begin{aligned}
j_{L H}= & -\frac{4 \pi \varepsilon_{0}^{2} m_{e}^{2}}{e^{3} \ln \Lambda} \int d v_{\|} D_{q l}\left(v_{\|}\right) \frac{\partial f_{e}\left(v_{\|}\right)}{\partial v_{\|}} \frac{4 v_{\|}^{3}}{5+Z} \\
& \times\left(\mu-\frac{1+Z / 2+3 \mu^{2} / 2}{3+Z} \frac{v_{\|}^{2}}{v_{r}^{2}}\right)
\end{aligned}
$$

where $Z$ is the effective ion charge, $\mu=-1$ for cooperational current drive and $\mu=+1$ for current drive into an opposing field. It is often necessary to iterate between TRANSP and the LHCD package for the field and current at the time that the LHCD package is called upon for information about current.

The LHCD package combined with the Brambilla coupling calculation is used in conjunction with the TRANSP code to obtain the driven current in a dynamic case.

\subsection{Transport model}

Since ion energy transport drops to roughly the neoclassical level in RS discharges, the ion thermal diffusivity model is assumed in terms of neoclassical transport enhanced by $\eta_{i}$ turbulence which is responsible for the transport outside the shear reversal region. The neoclassical ion thermal diffusivity is given by [24]

$$
\begin{aligned}
\chi_{i}^{n e o}= & n_{i} \varepsilon^{1 / 2}\left(\rho_{i p} / \tau_{i i}\right) K_{2}^{(0)}\left(\frac{K / 0.06}{1+a_{2} \mu_{* i}^{1 / 2}}+b_{2} \mu_{* i}\right. \\
& \left.+\frac{\left(c_{2}^{2} / b_{2}\right) \mu_{* i} \varepsilon^{3 / 2}}{1+c_{2} \mu_{* i} \varepsilon^{3 / 2}} H F\right)
\end{aligned}
$$

where $\varepsilon(\equiv r / R)$ is the reverse aspect ratio, $\rho_{i p}$ is the ion poloidal gyro-radius and

$$
\begin{aligned}
\mu_{* i}= & (1+1.54 \alpha) \nu_{* i i} \\
K= & {\left[0.66(1+1.54 \alpha)+\left(1.88 \varepsilon^{1 / 2}-1.54 \varepsilon\right)\right.} \\
& \times(1+3.75 \alpha)]\left\langle B_{0}^{2} / B^{2}\right\rangle \\
H= & 1+1.33 \alpha(1+0.60 \alpha) /(1+1.79 \alpha) \\
F= & 0.5 \varepsilon^{-1 / 2}\left(\left\langle B_{0}^{2} / B^{2}\right\rangle-\left\langle B^{2} / B_{0}^{2}\right\rangle^{-1}\right) .
\end{aligned}
$$

Here $\alpha=Z_{I}^{2} n_{I} / Z_{i}^{2} n_{i}$ with $Z_{I}\left(Z_{i}\right)$ the impurity (main ion) charge number. The other constants in Eq. (3) can be found in Ref. [24]. When $\eta_{i}$ (三 $\left.d \ln T_{i} / d \ln n\right)$ exceeds a threshold value $\eta_{i c}\left(\eta_{i c}=1.5\right.$ in the simulation), the ion thermal diffusivity is enhanced by the ion temperature gradient driven turbulence transport with the thermal diffusivity given by Ref. [25] as

$$
\begin{aligned}
\chi_{i}^{e n h}= & {\left[(\pi / 2) \ln \left(1+\eta_{i}\right)\right]^{2}\left[\left(1+\eta_{i}\right) / \tau\right]^{2} } \\
& \times\left(k_{0 y} \rho_{s}\right)\left(\rho_{s}^{2} c_{s} / L_{s}\right)
\end{aligned}
$$

where $\tau=T_{e} / T_{i}, \rho_{s}=c_{s} / \omega_{c i}, c_{s}$ is the sound speed and $L_{s}$ is the shear length.

In the enhanced confinement study in ASDEX, it has been assumed that ion energy transport is due to the neoclassical model combined with the anomalous transport from $\eta_{i}$ mode. This transport model qualitatively and quantitatively explains all ion energy balance features for both broad and peaked density profiles [26].

The electron energy transport is based on the Rebut-Lallia-Watkins (RLW) model [27]. In this model, it is assumed that turbulence in the magnetic topology, which is strongly dependent upon the shear of the field lines, is responsible for the anomalous transport. From heuristic and dimensional arguments, this model predicts the existence of a critical electron temperature gradient $\Delta T_{e c}$ such that the electron heat flow is given by

$$
\begin{aligned}
q_{e}= & -n_{e} \chi_{e}^{R L W} \nabla T_{e}\left(1-\left|\frac{\nabla T_{e c}}{\nabla T_{e}}\right|\right) \\
& \times H\left(\nabla T_{e}-\nabla T_{e c}\right) H(\nabla q)
\end{aligned}
$$

where $H$ is the Heaviside step function and transport is assumed to be neoclassical when $\left|\nabla T_{e}\right|<\left|\nabla T_{e c}\right|$ or $\nabla q<0$. The expressions for $\chi_{e}^{R L W}$ and $\nabla T_{e c}$ are [28] as follows:

$$
\begin{aligned}
\chi_{e}^{R L W}= & 0.5 c^{2} \sqrt{\mu_{0} m_{i}}\left(1-\sqrt{\frac{r}{R}}\right) \sqrt{1+Z_{e f f}} \\
& \times\left(\frac{\nabla T_{e}}{T_{e}}+2 \frac{\nabla n_{e}}{n_{e}}\right) \sqrt{\frac{T_{e}}{T_{i}}}\left(q^{2} / \nabla q B_{T} R^{1 / 2}\right) \\
\nabla T_{e c}= & 0.06 \sqrt{\frac{\eta j B_{T}^{3}}{n_{e} T_{e}^{1 / 2}}} \frac{1}{q} \sqrt{\frac{e^{2}}{\mu_{0} m_{e}^{1 / 2}}}
\end{aligned}
$$

where $\eta, j$ are the plasma resistivity and current density, respectively, and $B_{T}$ is the magnetic field. Moreover, the ion diffusivity

$\chi_{i}^{R L W}=2 \chi_{e}^{R L W}\left[Z_{i} / \sqrt{\left(1+Z_{e f f}\right)}\right] \sqrt{T_{e} / T_{i}}$

where $Z_{i}$ and $Z_{\text {eff }}$ are the ion charge and the plasma effective charge, respectively. The simulation for the JET ohmic and L mode discharges covering a quite 
wide range of plasma parameters showed that the RLW model emerges as rather good and complete $[29,30]$.

The RLW model is a gyro-Bohm model for both electrons and ions. Rosenbluth and co-workers modified the ratio $\chi_{i} / \chi_{e}$ of the standard RLW model to give ions a Bohm-like scaling [31],

$$
\begin{aligned}
\chi_{i}^{R L W} / \chi_{e}^{R L W}= & 0.6\left[Z_{i} / \sqrt{\left(1+Z_{\text {eff }}\right)}\right]\left[2 T_{e} /\left(T_{e}+T_{i}\right)\right] \\
& \times R B_{T} / \sqrt{\left(T_{e}+T_{i}\right)}
\end{aligned}
$$

and this can explain many experimental features of TFTR discharges as well [31].

In modelling RS operation for HL-2A, the modified RLW model $\left(\chi_{i}^{R L W}, \chi_{e}^{R L W}\right)$ is used during the non-RS phase. During the RS phase, since the electron heat flow is magnetic shear dependent in the RLW model, $\chi_{e}^{R L W}$ is used as the electron diffusivity, and neoclassical transport enhanced by $\eta_{i}$ turbulence $\left(\chi_{i}^{n e o}+\chi_{i}^{e n h}\right)$ is used as the ion diffusivity. As the electron and ion thermal diffusivities are simultaneously reduced in the central enhanced confinement region [10], in the RS region the electron diffusivity is adjusted to a similar level as for neoclassical ion transport by an empirical factor.

In the TRANSP simulation the electron density is prescribed. To give a margin for density control, target plasmas with different density profiles are assumed in the computation of sustained RS operation.

Recently a mixed Bohm and gyro-Bohm transport model is used to model the advanced tokamak discharges with the magnetic shear effect and/or the $E \times B$ effect incorporated into it explicitly [32-34]. The successful modelling of the formation and evolution of enhanced confinement regimes showed that the shear effects play a significant role in improving confinement in the core plasma region. In this article the mixed theory model described earlier, which has been validated for some experiments, is used to predict advanced operation scenarios in a new tokamak. Despite the fact that the magnetic shear effect does not enter explicitly into the ion transport model, some self-consistent results on the formation and maintenance of both reversed shear and ITB are obtained, showing that magnetic shear is relevant to the suppression of anomalous transport in high performance plasmas.

\subsection{Sustainment of RS discharges}

The target plasma is maintained by means of a 2.0 MW neutral beam (1.5 MW co-injection and

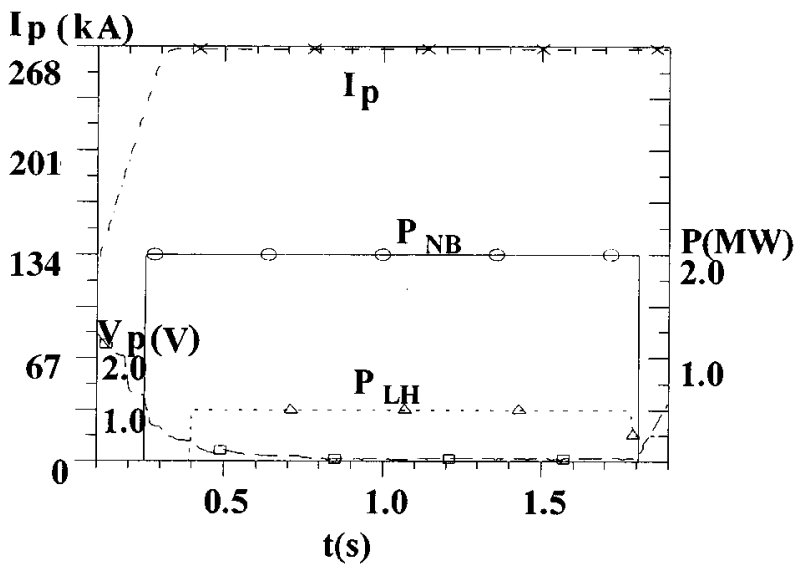

Figure 4. Waveforms of plasma current $I_{p}$, loop voltage $V_{p}$, NBI power $P_{N B}$ and LH wave power $P_{L H}$.

0.5 MW counter-injection) injected into an ohmic heating discharge (Fig. 4) with modest peaking density profile $\left(n_{e}(0) /\left\langle n_{e}\right\rangle=1.86, \bar{n}_{e}=2.32 \times\right.$ $\left.10^{19} \mathrm{~m}^{-3}\right)$. Lower hybrid wave power of $0.5-0.8 \mathrm{MW}$ at $2.45 \mathrm{GHz}$ is used for profile control. When a $0.5 \mathrm{MW} \mathrm{LH}$ wave is injected into a discharge of $265 \mathrm{kA}$, various driven current profiles are produced for different phasings $\left(\Delta \phi=60,90,120,150^{\circ}\right)$. We can group the current drive scenario into two categories: one is off-axis current drive that is favourable to magnetic shear reversal and the other in which the LH wave penetrates to the plasma centre.

With the phasing $\Delta \phi=90^{\circ}$, the profile of $\mathrm{LH}$ wave driven current is peaked off-axis with the peak of the current density located at $x \approx 0.6$. After a transitional phase that lasts about $0.35 \mathrm{~s}$, the driven current profile retains nearly the same shape during the temporal evolution (Fig. 5(a)). By using the off-axis LHCD combined with the effect of bootstrap current and beam driven current, a steady state RS discharge is formed and sustained with $\chi_{\min } \approx 0.6$ and $q_{\text {min }} \approx 2.8$ (Fig. 5(b)) until the LH power is turned off. Meanwhile the pressure profile of the RS plasma is flattened $(p(0) /\langle p\rangle=3.0-3.2)$. This RS discharge has achieved nearly a fully non-inductive current drive (Fig. 6) with a bootstrap current fraction of $\sim 23 \%$ of the total plasma current and a current drive efficiency $\eta_{C D} \approx 1.0 \times 10^{19} \mathrm{~A} / \mathrm{W} \mathrm{m}^{2}$ for the $\mathrm{LH}$ wave driven current, and the plasma loop voltage is nearly zero during the sustained RS phase (Fig. 4).

To examine the robustness of the sustainable RS scenario, we perform the RS discharge modelling for the following different target plasmas:

(a) Target (i), approximately balanced beam injection (1.0 MW co-injection and 0.8 MW counter- 

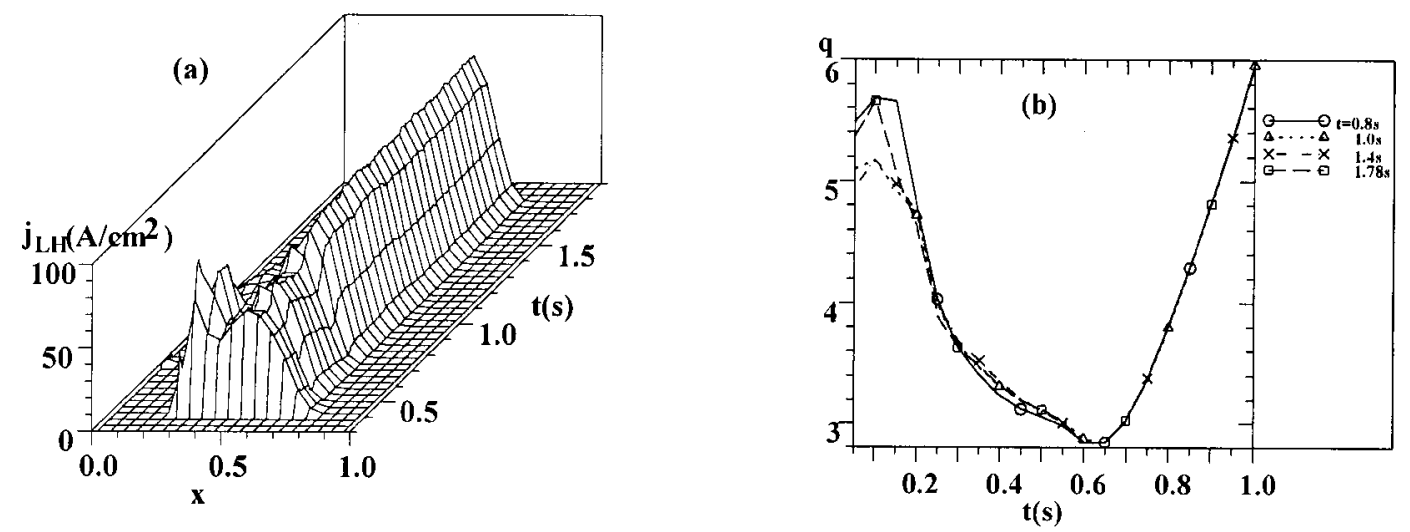

Figure 5. (a) Temporal evolution of LH wave driven current profile and (b) $q$ profiles at different times for a sustained RS discharge with $I_{p}=265 \mathrm{kA}$.
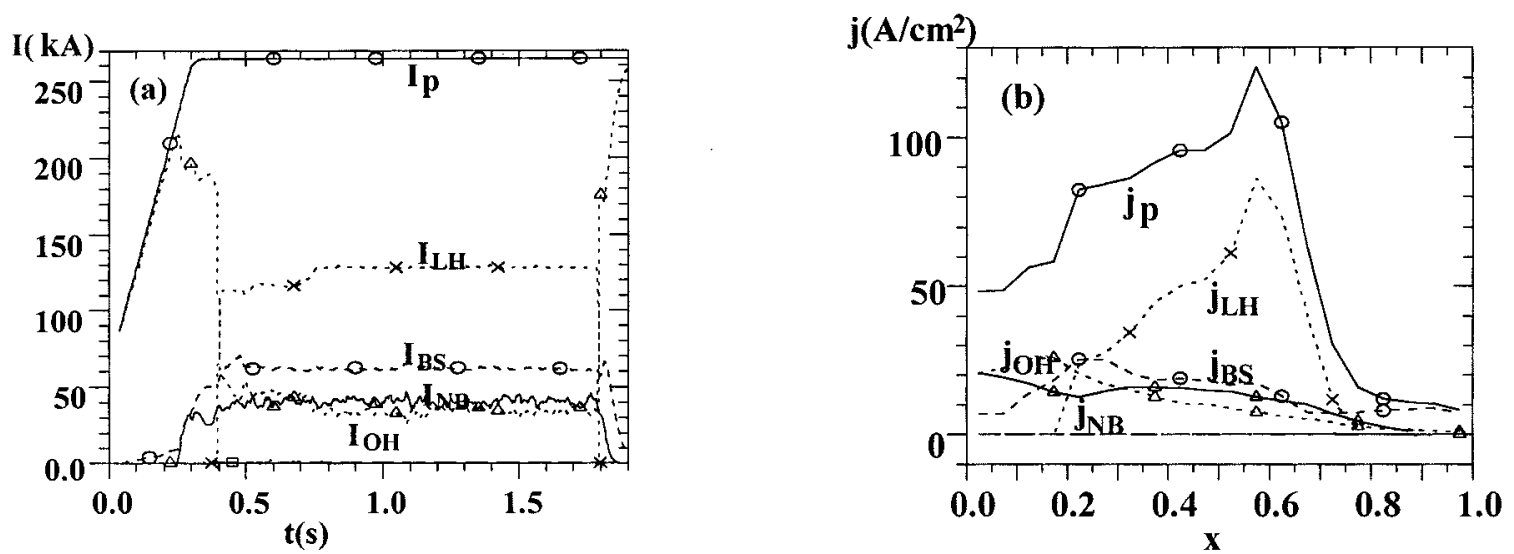

Figure 6. (a) Waveforms of plasma current $I_{p}$, LH wave driven current $I_{L H}$, bootstrap current $I_{B S}$, NBI driven current $I_{N B}$ and ohmic current $I_{O H}$ and (b) their profiles at $t=1.0 \mathrm{~s}$ for a sustained RS discharge.

injection) produces only a small beam driven current $I_{N B} \sim 10 \mathrm{kA}$ compared with $I_{N B} \sim$ $45 \mathrm{kA}$ in the standard target plasma;

(b) Target (ii), the peaking factor of the density profile $n_{e}(0) /\left\langle n_{e}\right\rangle$ increases by $\sim 20 \%$ by changing the density profile;

(c) Target (iii), the total plasma density increases by $10 \%$.

In all these cases nearly the same LH wave driven current profiles as in the standard case are produced during the steady state phase and sustained RS discharges are achieved. Nevertheless, when the plasma current increases to $I_{p}=300 \mathrm{kA}$ the $\mathrm{LH}$ wave driven current profile is centred with higher current drive efficiency $\left(\eta_{C D} \approx(1.2-1.8) \times 10^{19} \mathrm{~A} / \mathrm{W} \mathrm{m}^{2}\right)$ after the transitional phase (Fig. 7(a)), indicating that the
LH wave penetrates into the plasma centre. In this case the $q$ profile evolves approaching $q_{\min }<1.0$ and $x_{\min } \approx 0.2$ (Fig. $7(\mathrm{~b})$ ), and the RS discharge would not be sustained.

If the LH wave phase velocity is higher than 3.5 times the electron thermal velocity, there are too few velocity-resonant electrons to carry driven current density comparable with the ohmic current density. The corresponding required condition for current drive is

$n_{\|}=\frac{k_{\|} c}{\omega} \geq \frac{6.5}{\sqrt{T_{e}[\mathrm{keV}]}}$

where $k_{\|}$is the wave vector component parallel to the magnetic field. In the simulated RS discharges, it turns out $T_{e 0} \approx 1.4 \mathrm{keV}\left(T_{i 0} \approx 2.8 \mathrm{keV}\right)$ with $\bar{n}_{e}=$ $2.32 \times 10^{19} \mathrm{~m}^{-3}$. In such conditions there is a spectral 

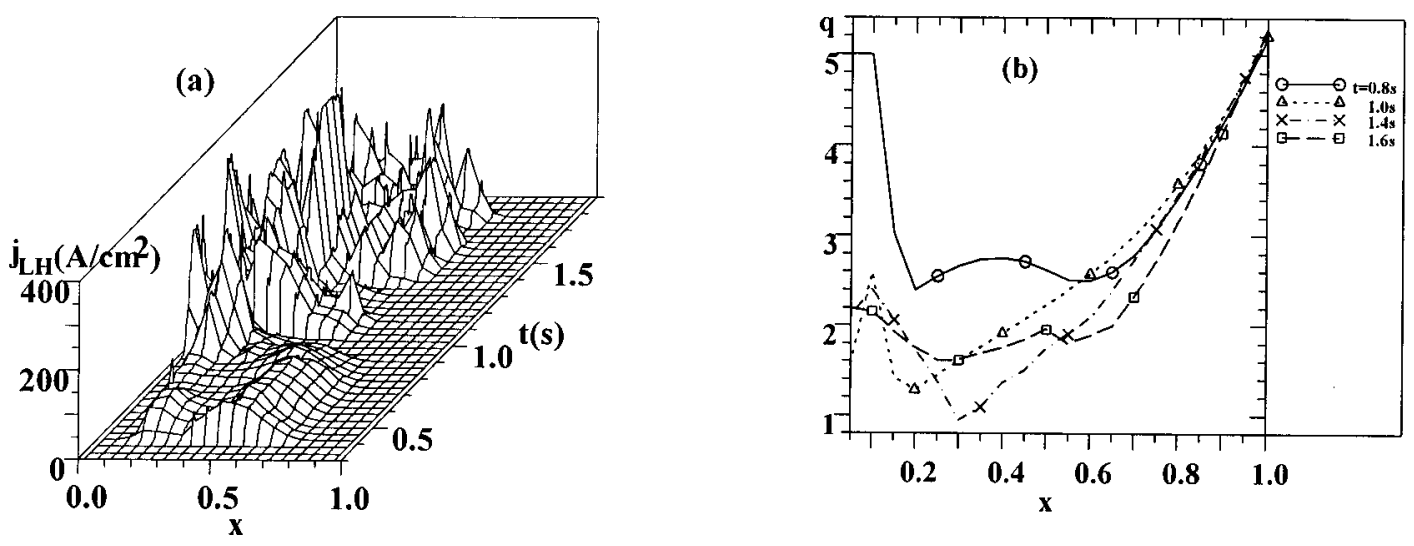

Figure 7. (a) Temporal evolution of the LH wave driven current profile and (b) $q$ profiles at different times for a discharge with $I_{p}=300 \mathrm{kA}$.

gap between the parallel LH wave phase velocity and the electron thermal velocity. To achieve off-axis LH wave power deposition in the weak damping regime, we rely on the $n_{\|}$upshift and on multiple reflections of the wave in the plasma. The $n_{\|}$upshift effect can be estimated from the toroidal axis symmetry and dispersion relation yielding [22]

$k_{\|} \leq k_{\| 0} \frac{R_{0} / R}{1-\left(B_{p} / B_{T}\right)\left(\omega_{p e} / \omega\right) / S^{1 / 2}}$

where

$S=1+\frac{\omega_{p e}^{2}}{\omega_{c e}^{2}}-\sum_{j} \frac{\omega_{p i, j}^{2}}{\omega^{2}}$

$k_{\| 0}$ and $R_{0}$ refer to the $k_{\phi}$ launched at the antenna $R_{0}, \omega_{p e}$ and $\omega_{p i}$ are the electron and ion plasma frequency, respectively, and $\omega_{c e}$ is the electron gyrofrequency.

During reduced magnetic field operation in Tore Supra, off-axis LH wave current drive in the weak damping regime is achieved due to the fact that the wave propagation domain in the $\left(n_{\|}, r\right)$ plane limits the maximum allowed $n_{\|}$upshift in the plasma core [6]. However, in the HL-2A RS operation achieved above the LH wave propagation domain is much wider (the LH wave propagation in HL-2A will be analysed elsewhere). To show the off-axis LH wave power deposition in HL-2A, we draw the electron Landau damping (ELD) limit (Eq. (10)) and the $n_{\|}$upshift boundary (Eq. (11)) in the $\left(n_{\|}, r\right)$ plane (Fig. 8). The LH wave absorption by strong ELD is bounded in the region above the ELD limit and below the $n_{\|}$upshift boundary. For the sustained RS operation obtained with $I_{p}=265 \mathrm{kA}$, it is shown in Fig. 8(a) that the spatial region of power deposition is limited to $0.5<x<0.85$, and it is off-axis.
Nevertheless, when the plasma current increases to $I_{p}=300 \mathrm{kA}$, the intersection between the upper $n_{\|}$ limit and the ELD limit is localized at $x \approx 0.1$. In this case the LH power can be deposited near the plasma centre, which would increase the central electron temperature allowing the LH wave to penetrate further into the centre. From the analysis above, it is concluded that the sensitivity of the LH driven current profile to the variation of total plasma current is due to the upshift factor imposed by the dispersion relation and the tokamak axis symmetry.

\subsection{Internal transport barrier}

In the sustained RS discharge modelled above the ITB is obtained and the plasma confinement is enhanced by $H_{89} \approx 1.8$ (Fig. 9). The ITB manifests itself by the higher gradient of ion temperature inside it (Fig. 10). The ITB is maintained stationary during the RS phase with the maximum of $\left|\nabla T_{i}\right|$ being near $r_{\text {min }}$. If we define the small region with higher $T_{i}$ gradient (say $>0.9 \max \left|\nabla T_{i}\right|$ ) to be the ITB layer, it is located around the shear reversal point as shown in Fig. 11. During the RS discharge the position of the ITB layer temporally evolves following the evolution of the shear reversal point (Fig. 11). In the simulation the theoretical transport model, the NBI heating and the LH current drive are all correlated with the plasma parameters, forming a strong nonlinear system, but the plasma temperature profiles and current profile evolve consistently, which shows that the system is self-consistent and the formation of the ITB is related to the magnetic shear reversal. 

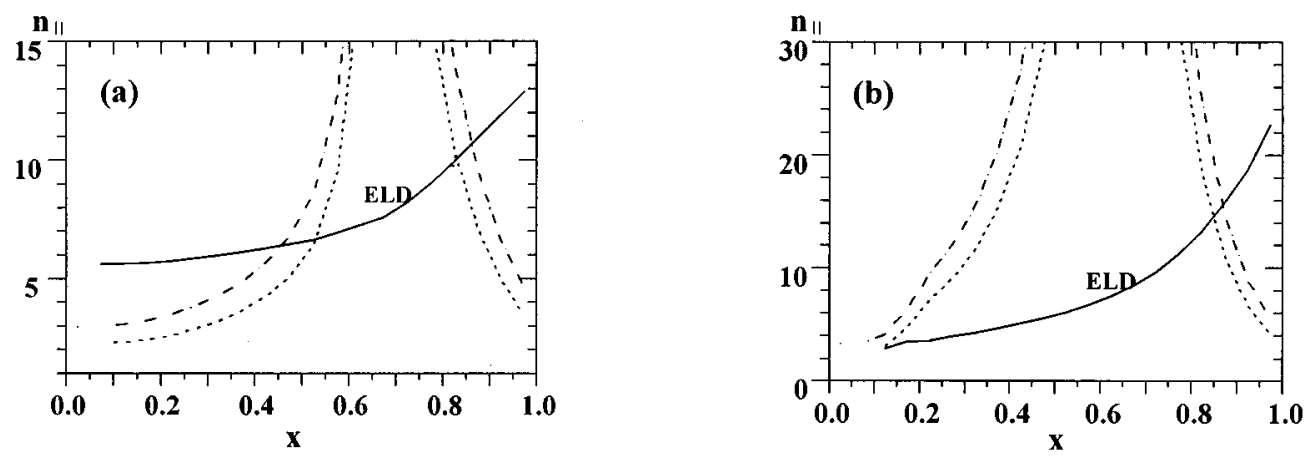

Figure 8. Region of LH power absorption by strong electron Landau damping: ELD limit (full curves); $n_{\|}$upshift boundary for $n_{\| c}-n_{H W}$ (dotted curves) and $n_{\| c}+n_{H W}$ (dashed curves), where $n_{H W}$ is the half width at half maximum of the $n_{\|}$spectrum. (a) $I_{p}=265 \mathrm{kA}$, (b) $I_{p}=300 \mathrm{kA}$.

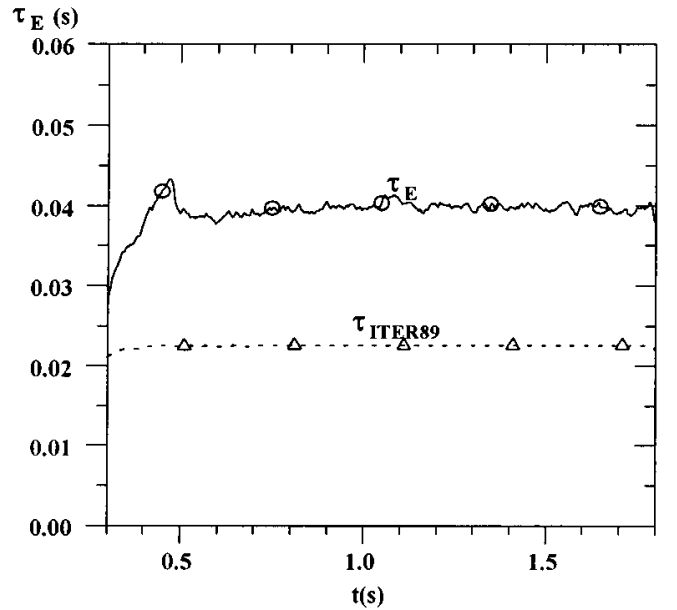

Figure 9. Energy confinement time $\tau_{E}$ and $\tau_{I T E R 89}$ versus time.

\section{Summary}

The TRANSP code, which has been benchmarked in many tokamak experiments, is used to model realistic reversed magnetic shear operation in HL-2A. We inject 2.5-3.0 MW of NBI power into a target plasma with gradually peaking density profile $\left(n_{e}(0) /\left\langle n_{e}\right\rangle=\right.$ 1.7-3.4). As plasma beta rises, RS configurations are formed with the minimum $q\left(q_{\text {min }}\right)$ evolving from $q_{\min }>3.0$ to $q_{\min }<2.0$ and $q_{\min }$ being localized at $r_{\text {min }} / a \approx 0.3$. An excessively peaked pressure profile (up to $p(0) /\langle p\rangle \geq 5.5$ ) and narrow negative shear region characterize these RS configurations.

To avoid excessive peaking pressure profile and sustain the RS operation towards steady state, offaxis current drive with an $\mathrm{LH}$ wave at $2.45 \mathrm{GHz}$ is

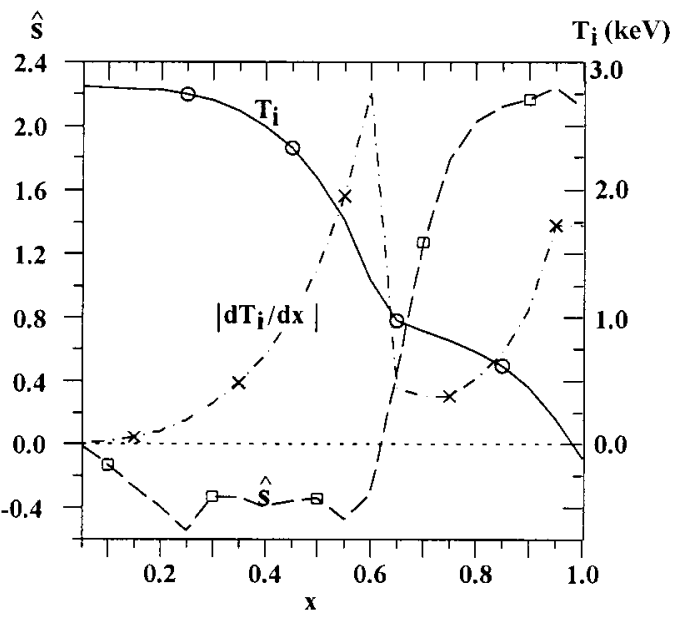

Figure 10. Ion temperature $T_{i}$, magnetic shear $\hat{s}$ and gradient of ion temperature $\left|d T_{i} / d x\right|$ versus $x$.

used to control the current profile. The target plasma is maintained by means of a $2.0 \mathrm{MW}$ neutral beam injected into an ohmic heating plasma with modest peaking density profiles $\left(n_{e}(0) /\left\langle n_{e}\right\rangle \approx 1.86\right)$. The LHCD simulation utilizes a toroidal ray tracing calculation and a parallel velocity Fokker-Planck calculation for the interaction of waves and particles. The LHCD package is used in conjunction with the TRANSP code to obtain the driven current in a dynamic case. As the ion energy transport drops to roughly the neoclassical level in RS discharges, the ion thermal diffusivity model is assumed in terms of neoclassical transport enhanced by $\eta_{i}$ turbulence that is responsible for the transport outside the shear reversal region. Electron energy transport is based on the RLW critical electron temperature gradient 


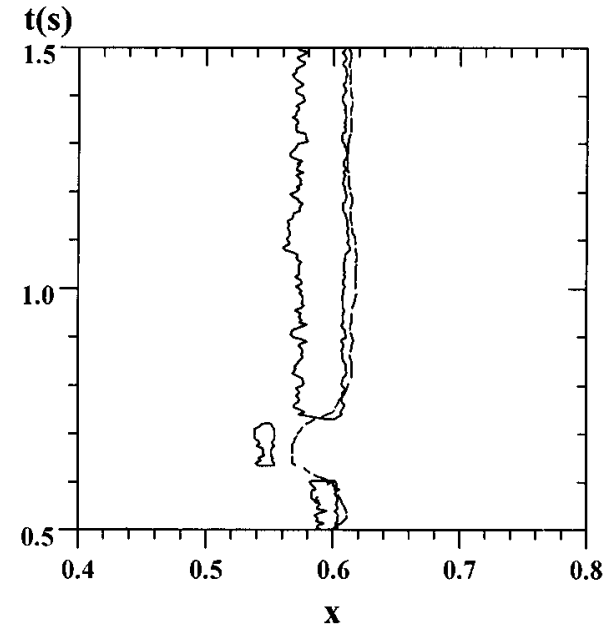

Figure 11. Temporal evolution of the position of the shear reversal point (where $\hat{s}=0$ ) (dashed line) and the position of the ITB layer (full line) during the RS phase.

model. When the $0.5 \mathrm{MW}$ LH wave power is injected, a stationary RS discharge is formed after a $0.35 \mathrm{~s}$ transitional phase. The stationary RS discharge with $r_{\text {min }} / a \approx 0.6$ and $q_{\text {min }} \approx 2.8$ is sustained until the LH wave power is turned off. During the stationary RS phase a self-consistent ITB appears on the ion temperature profile, implying enhanced energy confinement. The modelling results also show that when the LH wave current drive is used for current profile control, the RS configuration could be established with a wider shear reversal region and a broader pressure profile which is favourable to achieving a high beta limit $[17,18]$.

\section{Acknowledgements}

We are grateful to D.C. McCune and his group at PPPL for facilitating the TRANSP simulations. This work is supported by the National Natural Science Foundation of China under Grant No. 19889502 and the China Nuclear Science Foundation under Grant No. Y917C0315.

\section{References}

[1] Kessel, C., Manickam, J., Rewoldt, G., Tang, W.M., Phys. Rev. Lett. 72 (1994) 1212.

[2] Turnbull, A.D., Taylor, T.S., Lin-Liu, Y.R., St. John, H., Phys. Rev. Lett. 74 (1995) 718.

[3] Levinton, F.M., et al., Phys. Rev. Lett. 75 (1995) 4417.

[4] Strait, E.J., et al., Phys. Rev. Lett. 75 (1995) 4421.
[5] Koide, Y., JT-60 Team, Phys. Plasmas 4 (1997) 1623.

[6] Litaudon, X., et al., Plasma Phys. Control. Fusion 38 (1996) 1603.

[7] Söldner, F.X., JET Team, Plasma Phys. Control. Fusion 39 (1997) B353.

[8] Gormezano, C., et al., Phys. Rev. Lett. 80 (1998) 5544.

[9] Gruber, O., et al., Phys. Rev. Lett. 83 (1999) 1787.

[10] Ishida, S., JT-60 Team, Nucl. Fusion 39 (1999) 1211.

[11] Gao, Qingdi, HL-2A Team, "High Performance Operation of HL-2A and its Upgrade", paper presented at 1st Japan-China Workshop on Improved Performance in Toroidal Plasmas, Hefei, 1997.

[12] Budny, R.V., et al., Nucl. Fusion 35 (1995) 1497.

[13] Manickam, J., et al., in Fusion Energy 1996 (Proc. 16th Int. Conf. Montreal, 1996), Vol. 1, IAEA, Vienna (1996) 453.

[14] Chu, M.S., et al., Phys. Rev. Lett. 77 (1996) 2710.

[15] Huysmans, G.T.A., et al., in Controlled Fusion and Plasma Physics (Proc. 24th Eur. Conf. Berchtesgaden, 1997), Vol. 21A, Part I, European Physical Society, Geneva (1997) 21.

[16] Turnbull, A.D., et al., in Fusion Energy 1996 (Proc. 16th Int. Conf. Montreal, 1996), Vol. 2, IAEA, Vienna (1996) 509.

[17] Bondeson, A., Benda, M., Persson, M., Chu, M.S., Nucl. Fusion 37 (1997) 1419.

[18] Bonoli, P.T., Porkolab, M., Ramos, J.J., Nevins, W., Kessel, C., Plasma Phys. Control. Fusion 39 (1997) 223.

[19] Koide, Y., JT-60 Team, Phys. Plasmas 4 (1997) 1623.

[20] Gao, Qingdi, Budny, R.V., Zhang, Jinhua, Qu, Hongpeng, in Controlled Fusion and Plasma Physics (Proc. 26th Eur. Conf. Maastricht, 1999), Vol. 23J, European Physical Society, Geneva (1999) 525.

[21] Brambilla, M., Nucl. Fusion 16 (1976) 47.

[22] Ignat, D.W., Valeo, E.J., Jardin, S.C., Nucl. Fusion 34 (1994) 837.

[23] Karney, C.F.F., Fisch, N.J., Phys. Fluids 29 (1986) 180.

[24] Chang, C.S., Hinton, F.L., Phys. Fluids 29 (1986) 3314.

[25] Lee, G.S., Diamond, P.H., Phys. Fluids 29 (1986) 3291.

[26] Mertens, V., et al., Physics of Enhanced Confinement with Peaked and Broad Density Profiles, Rep. IPP-III/164, Max-Planck-Institut für Plasmaphysik, Garching (1990).

[27] Rebut, P.H., Lallia, P.P., Watkins, M.L., in Plasma Physics and Controlled Nuclear Fusion Research 1988 (Proc. 12th Int. Conf. Nice, 1988), Vol. 2, IAEA, Vienna (1989) 191.

[28] Rebut, P.H., Watkins, M.L., Gambler, D.J., 
Boucher, D., Phys. Fluids B 3 (1991) 2209.

[29] Corrigan, G., et al., in Controlled Fusion and Plasma Heating (Proc. 17th Eur. Conf. Amsterdam, 1990), Vol. 14B, Part II, European Physical Society, Geneva (1990) 801.

[30] Taroni, A., et al., in Plasma Physics and Controlled Nuclear Fusion Research 1990 (Proc. 13th Int. Conf. Washington, DC, 1990), Vol. 1, IAEA, Vienna (1991) 93.

[31] Rosenbluth, M.N., et al., in Plasma Physics and Controlled Nuclear Fusion Research 1994 (Proc. 15th Int. Conf. Seville, 1994), Vol. 3, IAEA, Vienna (1996) 517.
[32] Parail, V.V., et al., Nucl. Fusion 39 (1999) 429.

[33] Litaudon, X., et al., Plasma Phys. Control. Fusion 41 (1999) A733.

[34] Voitsekhovitch, I., et al., Phys. Plasmas 6 (1999) 4229 .

(Manuscript received 10 December 1999

Final manuscript accepted 11 August 2000)

E-mail address of Qingdi Gao: qgao@swip.ac.cn

Subject classification: F2, Ti; H1, Ti 